\title{
EPICYCLIC GEAR TRAIN SYNTHESIS
}

The synthesis of an epicyclic gear train is a difficult task that can be solved by different methods with significantly various computational time demands. This article describes effective approach to the two-linked epicyclic gear train synthesis. The method described in this article is based on the fundamental gear ratio calculation for each possible combination of gearbox external elements connected to the internal planetary structure.

Keywords: Epicyclic gear train, synthesis, Willis formula.

\section{Introduction}

The two-linked epicyclic gear train synthesis rests in the all possible kinematic structure creation and the selection of its best alternative.

The starting point of the calculation is the definition of gear ratios in the solved gearbox via selected method. The most common method for the ratio calculation is the drive resistance method, which generates the minimum gear ratio and the method of the maximum velocity, which generates the minimum gear ratio [1].

After defining the couple of ratios, the main task of the synthesis is to find the optimal kinematic structure of the epicyclic gear train via the analytical method using the specific features of the kinematic equations of the two-linked planetary gear trains [2].

\section{Single epicyclic gear kinematic equations}

The basic equation, which characterises the epicyclic gear train with 3 basic elements (sun gear $p$, ring gear $q$ and carrier $r$ ) and which has 2 degrees of freedom (DOF) is so called Willis formula

$\omega_{p}-\omega_{q} u_{v}=\omega_{r}\left(1-u_{v}\right)$

where: $\quad \omega_{\mathrm{p}}$ speed of the central wheel $p$ (sun gear), $\omega_{1}$ speed of the central wheel $q$ (ring gear),

$\omega_{\mathrm{r}}$ speed of the carrier $r$,

$u_{v}$ fundamental epicyclic gear ratio.

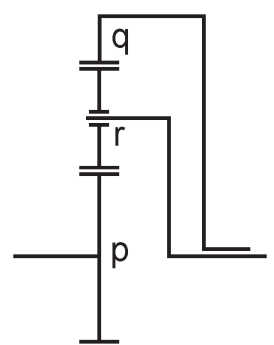

Fig. 1 Single epicyclic gear train with internal geared ring gear (Source: authors)

The fundamental epicyclic gear ratio is defined as

$u_{v}= \pm \frac{z_{q}}{z_{p}}=\frac{\omega_{p}}{\omega_{q}} \mid \omega_{r}=0$,

where: $\quad z_{\mathrm{q}}$ tooth number of the central wheel $q$, $z_{\mathrm{p}}$ tooth number of the central wheel $p$.

The sense of the gear ratio is negative if the central wheel $q$ has the internal gearing, which is the case of both of the twolinked simply epicyclic gear trains solved in this task (Fig. 3).

The Willis equation (1) has specifical properties considering the fundamental epicyclic gear ratio $u_{\mathrm{v}}$ as negative [3] and [4]:

- it is linear and homogeneous,

- the summary of its constant coefficients is zero: $1-u_{v}-\left(1-u_{v}\right)=0$

- the coefficient with the lowest absolute value is 1 and it is placed by the speed of the central wheel $p$,

\footnotetext{
* ${ }^{1}$ Frantisek Brumercik - ${ }^{1}$ Maria Tomasikova - ${ }^{2}$ Aleksander Nieoczym 
- the coefficient with the highest absolute value is $\left|1-u_{\mathrm{v}}\right|$ and it is placed by the speed of the central wheel $q$,

- the equation is valid for any epicyclic gear with three basic elements $(p, q, r)$ with two degrees of freedom.

Every step-gearbox based on epicyclic gears has following external elements:

- Input $A$,

- Output $X$,

- Braking elements $n$, which represents the connection of the internal gearbox element to the gearbox frame to obtain the $\mathrm{DOF}=1$. The number of braking elements corresponds with the number of gear ratios [5] (excluding the ratio $i_{\mathrm{AX}}=1$ direct drive)

Thus, the preferred ratio is shifted by the activating of the proper braking element $(n=1,2, \ldots)$.

The gear ratio between the input and output is defined as [6]

$$
i_{A X}=\frac{\omega_{A}}{\omega_{X}}
$$

where: $\omega_{\mathrm{A}}$ speed of the input $A$,

$\omega_{\mathrm{X}}$ speed of the output $X$.

Consider the input $A$ is connected to the central gear $p$, the output $X$ is connected to the central gear $q$ and the stopped braking element $n$ is the carrier $r$ (Fig. 2).

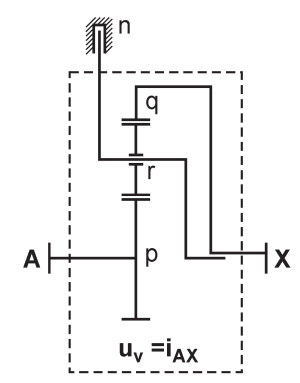

Fig. 2 Single epicyclic gear in the basic connection (Source: authors)

Then, the fundamental epicyclic gear ratio $u_{\mathrm{v}}$ is the same as the gear ratio between the input and output of the mechanism $i_{\mathrm{AX}}$ and the Willis formula can be represented also with the help of input and output speeds of the epicyclic gearbox and the gear ratio between input and output $i_{\mathrm{AX}}$ as

$\omega_{A}-\omega_{X} i_{A X}=\omega_{n}\left(1-i_{A X}\right)$,

where: $\omega_{\mathrm{A}}$ speed of the input - central wheel $p$, $\omega_{\mathrm{X}}$ speed of the output - central wheel $q$, $\omega_{\mathrm{n}}$ speed of the braking element - carrier $r$, $i_{\mathrm{AX}}$ desired gear ratio.
Each simple planetary gear (Fig. 1) can produce 6 gear ratios dependent on the motion state [7] and [8]. The motion state is described by the graphic expression $\left(\left.p \rightarrow q\right|_{r=0}\right)$. The input element is placed on the first place $(p)$, the arrow symbol $(\rightarrow)$ is expressing the power flow to the output element behind the arrow $(q)$, whereby the stopped element $(r)$ is described behind the vertical line $(\mid r=0)$. The example of the motion state is matching the gear structure in Fig. 2.

The relations $i_{A X}=f\left(u_{v}\right)$ and $u_{v}=f\left(i_{A X}\right)$ are shown in Tables 1 and 2.

Relations between the gear ratio $i_{\mathrm{AX}}$ and the fundamental ratio $u_{\mathrm{v}}$

Table 1

\begin{tabular}{|c|c|c|c|}
\hline Motion state & $i_{A X}=f\left(u_{v}\right)$ & Motion state & $i_{A X}=f\left(u_{v}\right)$ \\
\hline$\left.p \rightarrow q\right|_{r=0}$ & $i_{A X}=u_{v}$ & $\left.q \rightarrow p\right|_{r=0}$ & $i_{A X}=\frac{1}{u_{v}}$ \\
\hline$\left.p \rightarrow r\right|_{q=0}$ & $i_{A X}=1-u_{v}$ & $\left.r \rightarrow p\right|_{q=0}$ & $i_{A X}=\frac{1}{1-u_{v}}$ \\
\hline$\left.q \rightarrow r\right|_{p=0}$ & $i_{A X}=\frac{u_{v}-1}{u_{v}}$ & $\left.r \rightarrow q\right|_{p=0}$ & $i_{A X}=\frac{u_{v}}{u_{v}-1}$ \\
\hline
\end{tabular}

Relations between fundamental ratio $u_{\mathrm{v}}$ and the gear ratio $i_{\mathrm{AX}}$ Table 2

\begin{tabular}{|c|c|c|c|}
\hline Motion state & $u_{v}=f\left(i_{A X}\right)$ & Motion state & $u_{v}=f\left(i_{A X}\right)$ \\
\hline$\left.p \rightarrow q\right|_{r=0}$ & $i_{A X}=u_{v}$ & $\left.q \rightarrow p\right|_{r=0}$ & $u_{v}=\frac{1}{i_{A X}}$ \\
\hline$\left.p \rightarrow r\right|_{q=0}$ & $u_{v}=1-i_{A X}$ & $\left.r \rightarrow p\right|_{q=0}$ & $u_{v}=\frac{i_{A X}-1}{i_{A X}}$ \\
\hline$\left.q \rightarrow r\right|_{p=0}$ & $u_{v}=\frac{1}{1-i_{A X}}$ & $\left.r \rightarrow q\right|_{p=0}$ & $u_{v}=\frac{i_{A X}}{i_{A X}-1}$ \\
\hline
\end{tabular}

\section{Two-linked epicyclic gear kinematic equations}

If the number of desired non-direct gear ratios is 2, there are 4 external elements needed to obtain the desired ratios [9]. The structure of such gearbox without connections between external and internal element connections is shown in Fig. 3.

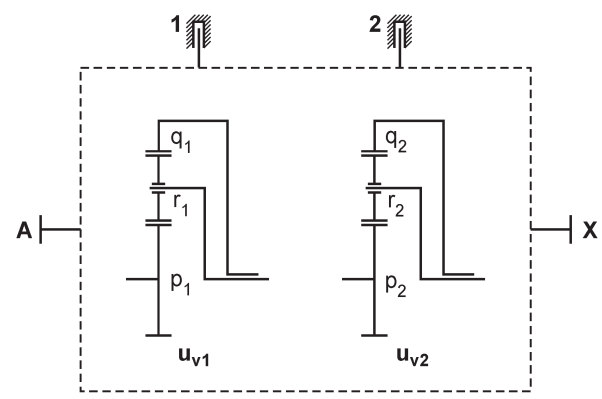

Fig. 3. Two-linked epicyclic gear - external and internal elements (Source: authors) 
By the consideration of the upper described assumptions defined for single epicyclic gear, two basic equations describing the relations among the elements $A, X, 1$ and 2 respectively can be written:

$$
\begin{aligned}
& \omega_{A}-\omega_{X} i_{A X 1}=\omega_{1}\left(1-i_{A X 1}\right), \\
& \omega_{A}-\omega_{X} i_{A X 2}=\omega_{1}\left(1-i_{A X 2}\right)
\end{aligned}
$$

These are so called basic equations describing the relations between the speeds of the external elements $A, X$ and 1 , or $A, X$ and 2 considering that the desired gear ratio can be achieved with the help of one planetary gear set using the described external elements connected to the internal planetary gear elements $\left(p_{1}\right.$, $\left.q_{1}, r_{1}, p_{2}, q_{2}, r_{2}\right)$ in so far unknown structure [10].

The other two so called derived equations can be obtained using the two basic equations (5). The product of this mathematical notation is the relations between the elements $A, 1$ and 2, or $X$, 1 and 2 respectively. The equations can be written in the form of

$$
\begin{aligned}
& \frac{\omega_{A}-\left(1-i_{A X 1}\right) \omega_{1}}{i_{A X 1}}=\frac{\omega_{A}-\left(1-i_{A X 2}\right) \omega_{2}}{i_{A X 2}} \\
& -i_{A X 1} \omega_{X}-\left(1-i_{A X 1}\right) \omega_{1}=-i_{A X 2} \omega_{X}-\left(1-i_{A X 2}\right) \omega_{2}
\end{aligned}
$$

After mathematical modification of the derived equations (6), they can be written as

$\omega_{A}-i_{A 12} \omega_{X}=\omega_{1}\left(1-i_{A 12}\right)$,

$\omega_{A}-i_{X 12} \omega_{X}=\omega_{1}\left(1-i_{X 12}\right)$

where: $i_{A 12}=\frac{-\left(1-i_{A X 1}\right) i_{A X 2}}{i_{A X 1}-i_{A X 2}}, i_{X 12}=\frac{-\left(1-i_{A X 1}\right)}{i_{A X 1}-i_{A X 2}}$

The ratios marked $i_{\mathrm{AX} 1}, i_{\mathrm{AX} 2}, i_{\mathrm{A} 12}, i_{\mathrm{X} 12}$ can be considered as so called general ratio $i_{\mathrm{q}}$, which will identify the internal structure of the mechanism in next calculations.

The general gear ratio $i_{\mathrm{q}}$ and the fundamental epicyclic gear ratio $u_{\mathrm{v}}$ are in mutual relation depending on connections between the external elements of the planetary gear train $(A, X, 1,2)$ and internal planetary elements of the planetary gear train $\left(p_{1}, q_{1}, r_{1}\right.$, $\left.p_{2}, q_{2}, r_{2}\right)$.

The relations $i_{q}=f\left(u_{v}\right)$ and $u_{v}=f\left(i_{A X}\right)$ derived from Tables 1 and 2 are shown in Tables 3 and 4.

Relations between the gear ratio $i_{\mathrm{q}}$ and the fundamental ratio $u_{\mathrm{v}}$

Table 3

\begin{tabular}{|c|c|c|c|}
\hline Motion state & $i_{q}=f\left(u_{v}\right)$ & Motion state & $i_{q}=f\left(u_{v}\right)$ \\
\hline$\left.p \rightarrow q\right|_{r=0}$ & $i_{q}=u_{v}$ & $\left.q \rightarrow p\right|_{r=0}$ & $i_{q}=\frac{1}{u_{v}}$ \\
\hline$\left.p \rightarrow r\right|_{q=0}$ & $i_{q}=1-u_{v}$ & $\left.r \rightarrow p\right|_{q=0}$ & $i_{q}=\frac{1}{1-u_{v}}$ \\
\hline$\left.q \rightarrow r\right|_{p=0}$ & $i_{q}=\frac{u_{v}-1}{u_{v}}$ & $\left.r \rightarrow q\right|_{p=0}$ & $i_{q}=\frac{u_{v}}{u_{v}-1}$ \\
\hline
\end{tabular}

The reciprocal table, which will be used by the synthesis, shows the relation between fundamental gear ratio of the simple epicyclic gear $u_{\mathrm{v}}$ as the function of the general gear ratio $i_{\mathrm{q}}$ (Table 2).

Relations between fundamental ratio $u_{v}$ and the gear ratio $i$ Table 4

\begin{tabular}{|c|c|c|c|}
\hline Motion state & $u_{v}=f\left(i_{q}\right)$ & Motion state & $u_{v}=f\left(i_{q}\right)$ \\
\hline$\left.p \rightarrow q\right|_{r=0}$ & $u_{v}=i_{q}$ & $\left.q \rightarrow p\right|_{r=0}$ & $u_{v}=\frac{1}{i_{q}}$ \\
\hline$\left.p \rightarrow r\right|_{q=0}$ & $u_{v}=1-i_{q}$ & $\left.r \rightarrow p\right|_{q=0}$ & $u_{v}=\frac{i_{q}-1}{i_{q}}$ \\
\hline$\left.q \rightarrow r\right|_{p=0}$ & $u_{v}=\frac{1}{1-i_{q}}$ & $\left.r \rightarrow q\right|_{p=0}$ & $u_{v}=\frac{i_{q}}{i_{q}-1}$ \\
\hline
\end{tabular}

\section{Synthesis of the two-linked epicyclic gear}

The equations in Table 4 can be used by finding the proper possibilities of fundamental ratio $u_{\mathrm{v}}$ of all possible combinations of connections of external elements to internal gear train elements $(A X 1, A X 2, A 12, X 12)$ using a simple algorithm, which calculates all the possible values of $u_{\mathrm{v}}$ that satisfy the specified criteria.

The most common criteria are the minimal planet gear bearings dimensions criteria $u_{v} \leq-1,3$ and the radial gearbox dimensions criteria $u_{v} \geq-4$.

The motion state of the mechanism with satisfactory $u_{\mathrm{v}}$ value defines the connections between the external elements of the planetary gear train $(A, X, n)$ and internal planetary elements of the planetary gear train $(p, q, r)$.

If two gear ratios have to be achieved, there is two-linked epicyclic gear train needed. The calculation of the fundamental ratio $u_{\mathrm{v}}$ has to be done for every mechanism that is defined by the equations (5) and (7). The result of the calculation is the matrix with $4 * 6=24$ values of the fundamental epicyclic gear ratios.

The number of the two-linked epicyclic gear trains valid for the desired gear ratios varies according to the number of usable mechanisms calculated by the equation

$C_{k}(n)=\left(\begin{array}{l}n \\ k\end{array}\right)=\frac{n !}{(n-k) ! k !}$

where: $C$ number of combinations of the $n$ elements taken $k$ times, $n$ number of basic and derived mechanism equations $k$ number of planetary gear train links

If each of the mechanisms $(A X 1, A X 2, A 12, X 12)$ satisfies the criteria for fundamental ratio $u_{v}$, there are

$C_{2}(4)=\frac{4 !}{(4-2) ! 2 !}=6$

combinations of possible two-linked epicyclic gear trains ( $A X 1$ AX2, AX1-A12, AX1-X12, AX2-A12, AX2-X12, A12-X12). 
The motion states of the two-linked simple epicyclic gears with the satisfactory $u_{\mathrm{v} 1}$ and $u_{\mathrm{v} 2}$ values define then the connections between the external elements of the planetary gear train $(A, X, 1$, $2)$ and internal elements of the planetary gear train $\left(p_{1}, q_{1}, r_{1}, p_{2}\right.$, $q_{2}, r_{2}$ ) shown in Fig. 3 .

\section{Conclusion}

The synthesis of two-linked epicyclic gears produces the planetary gearbox with two possible gear ratios shifted by braking of two control elements. The way how to connect the external and internal elements of the gearbox according the desired gear ratios is based on the fundamental gear ratio calculation for each possible combination of gearbox external elements connected to the internal planetary structure and the control of its value that defines their motion state. The final structure is a twolinked epicyclic gear train built-up from two of valid simple gear combinations [11 and 12].

\section{References}

[1] KOHAR, R., HRCEK, S., MEDVECKY, S.: Usage of Dynamic Analysis to Determine Force Interactions between Components of Rolling Bearings. Communications - Scientific Letters of the University of Zilina, vol. 14, No. 3, 2012, pp. 62-67.

[2] KAMPF, R., LIZBETIN, J., LIZBETINOVA, L: Requirements of a Transport System User. Communications - Scientific Letters of the University of Zilina, vol. 14, No. 4, 2012, pp. 106-108.

[3] DROZDZIEL, P., KRZYWONOS, L.: The Estimation of the Reliability of the First Daily Diesel Engine Start-up During its Operation in the Vehicle. Maintenance and Reliability, 1(41), 2009, pp. 4-10, ISSN 1507-2711.

[4] HANDRIK, M., VASKO, M., KOPAS, P., SAGA, M.: Effective Finite Element Solution and Post-processing for Wide Load Spectrum. Communications - Scientific Letters of the University of Zilina, vol. 16, No. 3A, 2014, pp. 19-26.

[5] SAGA, M., VASKO, M., KOPAS, P., JAKUBOVICOVA, L.: Numerical Algorithm for Beam Residual Stress Identification. Communications - Scientific Letters of the University of Zilina, vol. 16, No. 3A, 2014, pp. 13-18.

[6] DROZDZIEL, P., KOMSTA, H., KRZYWONOS, L.: An Analysis of Costs of Vehicle Repairs in a Transportation Company - Part I. Transport Problems, vol. 7, No. 3, 2012, pp. 67-75.

[7] DROZDZIEL, P., KOMSTA, H., KRZYWONOS, L.: An Analysis of Costs of Vehicle Repairs in a Transportation Company - Part II. Transport Problems, vol. 7, No. 4, 2012, pp. 7-11.

[8] SAPIETOVA, A., SAGA, M., NOVAK, P., JAKUBOVICOVA, L.: Multi-software Platform for Solving of Multibody Systems Synthesis. Communications - Scientific Letters of the University of Zilina, vol. 14, No. 3, 2012, pp. 43-48.

[9] DROZDZIEL, P., KRZYWONOS, L., MADLENAK, R., RYBICKA, I.: Selected Aspects of Analyses of Failure Rates of Active Safety Systems in Buses. Communications - Scientific Letters of the University of Zilina, vol. 16, No. 3, 2014, pp. 114-119.

[10] KOHAR, R., HRCEK, S.: Dynamic Analysis of a Rolling Bearing Cage with Respect to the Elastic Properties of the Cage for the Axial and Radial Load Cases. Communications - Scientific Letters of the University of Zilina, vol. 16, No. 3A, 2014, pp. 74-81.

[11] BUKOVA, B., BRUMERCIKOVA, E., MADLENAK, R.: Transport and e-commerce (in Slovak). Bratislava : Wolters Kluwer, 2014, 172 p., ISBN 978-80-8168-130-1.

[12] BUKOVA, B., BRUMERCIKOVA, E., KOLAROVSZKI, P.: Spedition and Logistics (in Slovak). Bratislava : Wolters Kluwer, 2014, 318 p. ISBN 978-80-554-0925-2. 